\title{
Failure of Y-27632 to improve the culture of adult human adipose-derived stem cells
}

This article was published in the following Dove Press journal:

Stem Cells and Cloning:Advances and Applications

7 January 2015

Number of times this article has been viewed

\author{
Nuno Jorge Lamas ${ }^{1-3}$ \\ Sofia C Serra ${ }^{1,2}$ \\ António J Salgado ${ }^{1,2}$ \\ Nuno Sousa ${ }^{1,2}$
}

'Life and Health Sciences Research Institute (ICVS), School of Health Sciences (ECS), University of Minho, Braga, Portugal; ${ }^{2}$ CVS/3B's-PT Government Associate Laboratory, Braga/Guimarães, Portugal; ${ }^{3}$ Clinical Pathology Department, Centro Hospitalar do Alto Ave (CHAA), EPE, Guimarães, Portugal

Correspondence: Nuno Sousa Life and Health Sciences Research Institute (ICVS), School of Health Sciences (ECS), University of Minho, Campus de Gualtar, Braga, Portugal Tel +35I 253604806 $\mathrm{Fax}+35$ I 253604809

Email njcsousa@ecsaude.uminho.pt
Abstract: Y-27632 is a well-known inhibitor of the Rho-associated coiled kinase (ROCK) and has been shown to significantly improve the culture of a variety of multipotent stem cell types. However, the effects of Y-27632 on the expansion of adult human adipose-derived stem cell (hADSC) cultures remain to be established. Here, we aimed to characterize the effects of Y-27632 on the culture of hADSCs. Adult hADSCs were isolated from subjects submitted to elective plastic surgery procedures and cultivated in vitro under optimized conditions. Our results show that the continuous supplementation of hADSC cultures with Y-27632 led to decreased numbers of cells and decreased global metabolic viability of hADSC cultures when compared with control conditions. This effect appeared to be dependent on the continuous presence of the drug and was shown to be concentration-dependent and significant for $10 \mu \mathrm{M}$ and $20 \mu \mathrm{M}$ of Y-27632. Moreover, the Y-27632-induced decrease in hADSC numbers was not linked to a block in global cell proliferation, as cell numbers consistently increased from the moment of plating until passaging. In addition, Y-27632 was not able to increase the number of hADSCs present in culture 24 hours after passaging. Taken together, our results suggest that, in contrast to other stem cell types, Y-27632 supplementation is not a suitable strategy to enhance hADSC culture expansion.

Keywords: human mesenchymal stem cells, human multipotent stromal cells (hMSCs), ROCK inhibitor, MTS assay

\section{Introduction}

Stem cells hold the capacity to self-renew and to differentiate into tissue-specific cell types. Over the last decades, the identification of different populations of stem cells has attracted the interest of the scientific community due to their enormous potential, including applications in the field of regenerative medicine and drug discovery. ${ }^{1-3}$ Strategies for efficient culture of these stem cells have long been pursued, rendering the procedures for stem cell culture less complex over time. ${ }^{4,5}$ A recently identified small molecule that has been proven to be particularly advantageous in stem cell culture is Y-27632, a selective inhibitor of the Rho-associated coiled kinase (ROCK). ${ }^{6,7}$ This compound has been demonstrated to display pleiotropic and wide-range positive effects across different cells types, namely human embryonic stem cells (hESCs) and human induced pluripotent stem cells (hiPSCs). ${ }^{6,7}$ Several reports have established that treating cultures of adult and human embryonic stem cells with Y-27632 considerably increases the post-thaw cell viability, cell attachment, and post-passaging viability, in addition to significant improvements in cellular proliferation. ${ }^{6-10}$ However, the influence of Y-27632 in the culture of adult human adipose-derived stem cells (hADSCs) 
has not yet been thoroughly assessed. The hADSCs constitute a population of adult stem cells easily isolated from body tissues containing fat. ${ }^{11,12}$ Similarly to other stem cell types, hADSCs retain the capacity to self-renew and to differentiate into diverse cell types, namely adipocytes, osteoblasts, chondrocytes, myocytes, cardiomyocytes, and neuron-like cells. ${ }^{12-14}$ Due to their differentiation potential, simplicity to isolate, and ability to proliferate in vitro, the scientific community consider the hADSCs as an increasingly attractive source of patient-specific stromal/stem cells. ${ }^{15-17}$

Here, we set out to investigate if the supplementation of hADSC cultures with Y-27632 would significantly improve the culture of this population of adult multipotent stromal/stem cells. Our results demonstrate a Y-27632-induced decrease in hADSC number and global metabolic viability upon continuous Y-27632 supplementation, in comparison with control conditions. The effect was shown to be concentration-dependent (significant effect for $10 \mu \mathrm{M}$ and $20 \mu \mathrm{M}$ of Y-27632) and also dependent on the continuous presence of the drug. Interestingly, cell numbers increased from the moment of plating until passaging, so that the Y-27632-induced decrease in hADSC numbers was not linked to a block in global cell proliferation. In addition, Y-27632 could not increase the number of hADSCs present in culture 24 hours after passaging. Together, our results suggest that Y-27632 supplementation alone is not an appropriate strategy to significantly improve the culture conditions of adult hADSCs.

\section{Materials and methods}

\section{Adult human adipose-derived} stem cells isolation

Adult hADSCs were kindly provided by Jeffrey M Gimble (Pennington Biomedical Research Center, Baton Rouge, LA, USA). Cells were isolated according to a protocol previously described by Dubois et al. ${ }^{18}$ Briefly, liposuction aspirates from subcutaneous adipose tissue were obtained from patients submitted to elective plastic surgery procedures. All donors gave their written informed consent. Tissues were then digested in a $0.1 \%$ collagenase type I solution (Worthington Biochemical Corporation, Lakewood, NJ, USA) pre-warmed to $37^{\circ} \mathrm{C}$ for 60 minutes. Afterward, the digested tissue was centrifuged for 5 minutes at 300-500 $g$ at room temperature. The supernatant, containing mature adipocytes, was aspirated. The pellet was identified as the stromal vascular fraction (SVF). The SVF was resuspended and plated in T225 flasks in Stromal Medium (Dulbecco's Modified Eagle's Medium [DMEM]/ Ham's F-12, 10\% fetal bovine serum [FBS; Hyclone, Logan,
UT, USA], $100 \mathrm{U}$ penicillin/100 $\mu \mathrm{g}$ streptomycin/0.25 $\mu \mathrm{g}$ Fungizone ${ }^{\circledR}$ ) at a density of $0.156 \mathrm{~mL}$ of tissue digest $/ \mathrm{cm}^{2}$ of surface area for expansion and culture. After reaching confluency, cells were passaged and kept in stromal medium.

\section{Adult human adipose-derived stem cells culture and survival/proliferation studies}

In this work, we used hADSCs previously cultured and cryopreserved between $\mathrm{p} 5$ and $\mathrm{p} 10$. All cell cultures were maintained in a humidified incubator at $37^{\circ} \mathrm{C}$ and $5 \% \mathrm{CO}_{2}$. After quickly thawing the vials in a $37^{\circ} \mathrm{C}$ water bath, cells were resuspended in culture medium containing Minimum Essential Medium $\alpha$ ( $\alpha$-MEM; Thermo Fisher Scientific, Waltham, MA, USA) supplemented with 10\% FBS (Thermo Fisher Scientific), 100 units/mL penicillin (Thermo Fisher Scientific), and $100 \mu \mathrm{g} / \mathrm{mL}$ streptomycin (Thermo Fisher Scientific ). Cells were then spun at $1,200 \mathrm{~g}$ for 5 minutes. The supernatant was gently aspirated and cells resuspended in the same culture medium and plated in a T75 flask and allowed to expand. Media was changed every 3-4 days. Upon confluency, cells were trypsinized and passaged to new T75 flasks or plated in 6-, 12-, or 24-well plates (Nunc; Thermo Fisher Scientific) for the survival/proliferation studies. These survival/proliferation studies were carried out using cells plated at two initial seeding densities $\left(1,000\right.$ and 5,000 cells $\left./ \mathrm{cm}^{2}\right)$. Y-27632 was added to the wells within the first 60 minutes after cell plating. Throughout the study, medium was changed in all conditions at selected time points. Cells were fixed at the desired time points ( 24 hours and 5 days post-plating). In each study, 2-3 replicate wells were used per studied condition.

\section{Y-27632}

The putative ROCK inhibitor Y-7632 was purchased from Abcam (ab120129; Cambridge, UK) and dissolved in deionized filtered water to yield a $10 \mathrm{mM}$ stock solution. Aliquots were prepared and stored at $-20^{\circ} \mathrm{C}$.

\section{Cell viability assessment}

Cell metabolic viability was assessed by performing MTS assays. The MTS 3-(4,5-dimethylthiazol-2-yl)-5-(3carboxymethoxyphenyl)-2-(4-sulfophenyl)-2H-tetrazolium test. The MTS test (Promega Corporation, Fitchburg, WI, USA) is a cell viability assay based on the bioreduction of the substrate (MTS) to a brown formazan product. After the desired cell culture period, the medium was aspirated and cells replenished with a new serum-free medium containing MTS in a 5:1 ratio and incubated at $37^{\circ} \mathrm{C}$ with $5 \%$ humidified 
$\mathrm{CO}_{2}$. Three hours post-incubation, $150 \mu \mathrm{L}$ of each sample were transferred to 96 -well plates $(n=3$ or 4 ) and optical density (OD) at $490 \mathrm{~nm}$ was determined using a Model 680 Microplate Reader (Bio-Rad Laboratories Inc., Hercules, CA, USA).

\section{Cell proliferation}

The cellular proliferation of hADSCs was determined by a colorimetric assay based on 5-bromo-2'-deoxyuridine (BrdU) incorporation (Hoffman-La Roche Ltd, Basel, Switzerland). Cells were incubated with BrdU over 48 hours. After the BrdU incubation period, an enzyme-linked immunosorbent assay (ELISA) test was performed according to the manufacturers recommended protocol. In the end, the OD was determined at $370 \mathrm{~nm}$ with a reference filter at $492 \mathrm{~nm}$ using an Infinite 200 PRO NanoQuant Reader (Tecan Group Ltd, Männedorf, Switzerland).

\section{Immunocytochemistry}

Adult hADSCs cultures were pre-fixed by adding one volume of cold $4 \%$ paraformaldehyde diluted in phosphatebuffered saline $1 \times($ PBS $1 \times / 4 \%$ PFA) for 2 minutes at room temperature. Then, cells were fixed with PBS $1 \times / 4 \%$ PFA for 30 minutes at $4^{\circ} \mathrm{C}$. After fixation, cells were washed with PBS $1 \times$ three times and then incubated in $0.1 \mu \mathrm{g} / \mathrm{mL}$ of red phalloidin (Sigma-Aldrich Co, St Louis, MO, USA) diluted in PBS $1 \times$ for 1 hour at room temperature. In the end, cells were again washed with PBS $1 \times$ and finally incubated in a solution containing $1 \mu \mathrm{g} / \mathrm{mL}$ 4',6-diamidino-2-phenylindole (DAPI; Thermo Fisher Scientific) for 20 minutes. Cells were washed again with PBS $1 \times$ and then imaged.

\section{Image acquisition and quantitative image analysis}

Image acquisition was performed using an IX-81 fluorescence microscope (Olympus Corporation, Tokyo, Japan). Seven representative fields of each well were selected in a blinded and systematic procedure using $10 \times$ magnification. Automated quantitative determination of DAPI-positive nuclei was performed using the Cell Scoring module of Metamorph NX software (Molecular Devices LLC, Sunnyvale, CA, USA). Positive cells were selectively identified when having clear signal intensity above local background. Intensity thresholds were set blinded to sample identity and in a given experiment, identical parameters were used in all images analyzed. The parameters were minimally adjusted across the different independent experiments.

\section{Statistical analysis}

All quantitative data were analyzed using GraphPad Prism for Windows (v5.00; GraphPad Software, San Diego, CA, USA). For each set of data, each condition was compared using one-way analysis of variance (ANOVA) statistical evaluation followed by Tukey's honestly significant difference (HSD) post hoc test. In cases involving only one time point and a two-group comparison, $P$-value was determined using Student's $t$-tests. Differences were considered to be significant when $P<0.05$.

\section{Results}

\section{The expansion of hADSCs is not enhanced in the presence of $\mathrm{Y}-27632$}

Several reports in the last decade have demonstrated that Y-27632 efficiently improves the culture conditions of a myriad of human stem cell types, including hESCs, hiPSCs, human mesenchymal stem cells (hMSCs) and human neuronal progenitor cells (hNPCs). ${ }^{6,7,9,10,19-23}$ However, the influence of Y-27632 in the culture of adult hADSCs remains to be firmly established. Here, we first aimed to assess if the presence of Y-27632 in the culture medium could improve the overall expansion of hADSCs. Human ADSCs p5 and p10 were grown in T75 flasks until confluency (Figure 1A). Afterward, cells were trypsinized and the resultant cell suspension recovered to be replated in 12-well plates. The pilot experiments performed showed that hADSC cultures seeded initially at the standard 5,000 cells $/ \mathrm{cm}^{2}$ seeding density would reach confluency after 5 days of continuous culture (control hADSC growth condition; Figure 1B). Thus, all subsequent expansion experiments were conducted until day 5 after hADSC plating. To evaluate the effect of Y-27632 in hADSCs, these adult stem cells were grown under continuous standard $10 \mu \mathrm{M}$ Y-27632 supplementation for 5 days (Y-27632 D0-D5); Y-27632 treatment for the first 24 hours followed by culture in regular growth conditions for the next 4 days (Y-27632 D0-D1); or growth in control conditions (control; Figure 1A). Two initial cell-seeding densities (1,000 and 5,000 cells $/ \mathrm{cm}^{2}$ ) were used (Figure 1A and B). During the 5-day period, a continuous increase in the number of hADSCs was registered across the three different medium supplementation conditions. At day 5, the cell cultures were fixed with PFA and stained for quantification of the number of hADSCs present in the culture. Using $10 \times$ magnification, seven representative fields of each well were then imaged in a blinded and systematic manner (Figure 2A). Automated quantitative image analysis of hADSCs present in the wells 
A

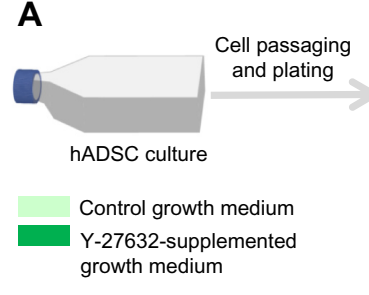

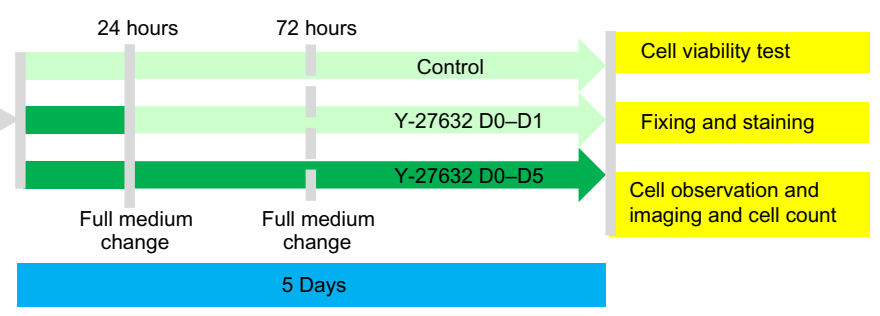
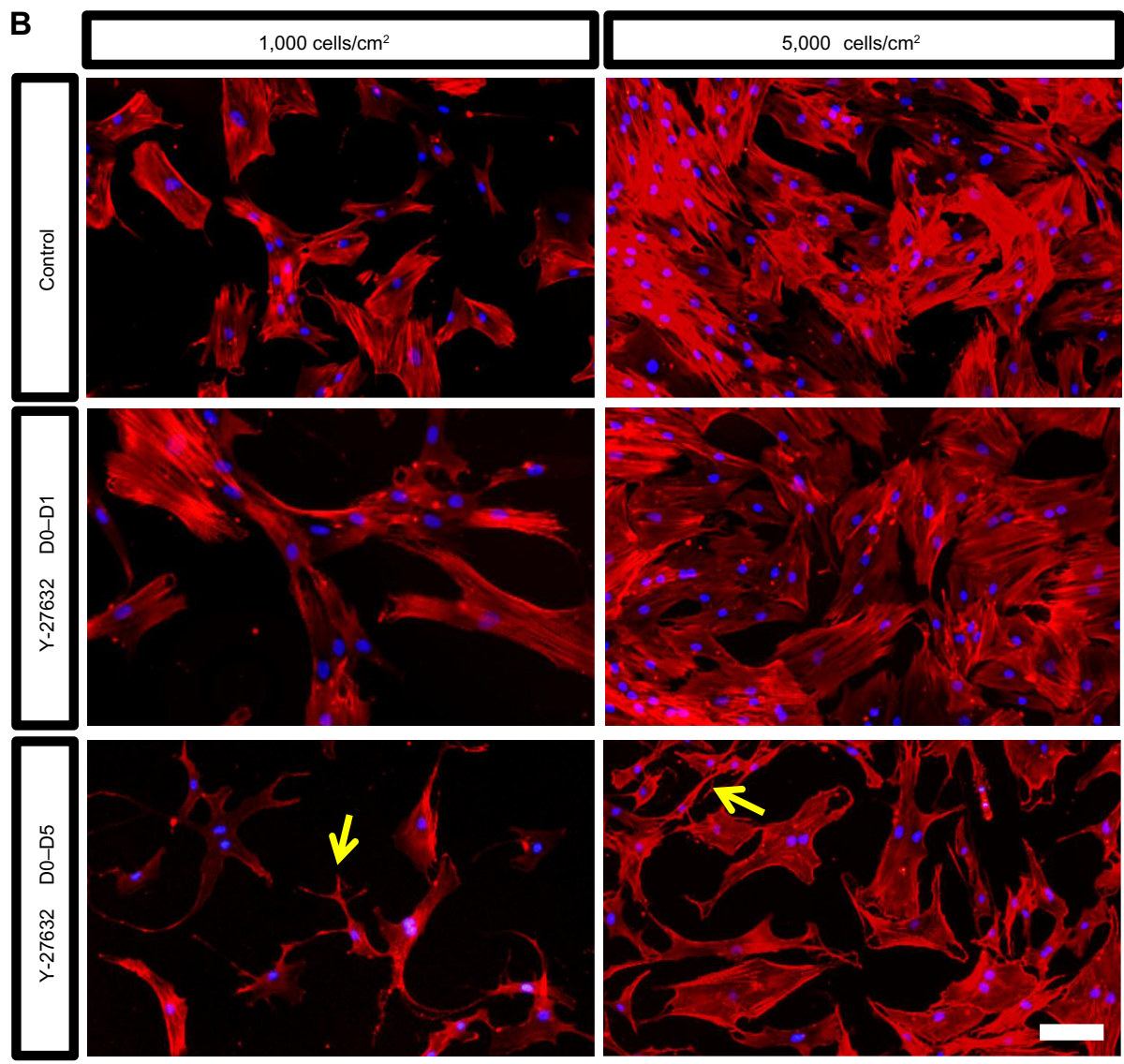

Figure I The continuous presence of $10 \mu \mathrm{M}$ Y-27632 did not lead to enhanced hADSC expansion.

Notes: (A) Diagram of the protocol used to study the effect of $Y-27632$ in hADSCs cultured over 5 days. Two initial seeding densities $\left(I \times 10^{3}\right.$ and $5 \times 10^{3}$ cells/ $\left./ \mathrm{cm}^{2}\right)$ were used for these studies. Cells were grown in control growth medium for 5 days (Control); Y-27632 treatment for the first 24 hours and then growth in control medium for the next 4 days (Y-27632 D0-DI); or continuously cultivated in the presence of $10 \mu$ M Y-27632 for 5 days (Y-27632 D0-D5). (B) Representative images of hADSC cultures after 5 days of continuous culture. None of the treatments could halt cellular proliferation, and therefore, cell numbers increased significantly from day I. Uninterrupted supplementation of hADSCs with $10 \mu \mathrm{M}$ Y-27632 led to a lower cell number at day 5 comparative to control conditions. A few cells grown under the continuous influence of Y-27632 evidenced a neuronal-like morphology after 5 days (yellow arrows), but failed to demonstrate robust expression of putative neuronal markers. Scale bar $=80 \mu \mathrm{m}$. Abbreviation: hADSCs, human adipose-derived stem cells.

was then performed using the Cell Scoring module of the Metamorph NX software (Figure 2B). After 5 days of cell culture, we registered similar numbers of hADSCs in the control and Y-27632 D0-D1 wells (Figures 1B, 2C, and 2D). On the other hand, Y-27632 D0-D5 wells had significantly decreased numbers of hADSCs when compared to the control and Y-27632 D0-D1 wells (Figures 1B, 2C, and 2D). The analysis of the global cell metabolic viability using the MTS test revealed significantly decreased OD in hADSCs grown continuously in the presence of Y-27632 (Y-27632 D0-D5; Figure 2E and F). These results were observed for both seeding densities tested (Figure 2C-F). Remarkably, the number of hADSCs present in culture was also diminished even if the Y-27632 treatment was continuously applied over 4 days from day 1 to day 5 (data not shown). Interestingly, at day 5, we observed that a few cells treated with Y-27632 over 5 days exhibited neuron-like morphology (Figure 1B; Y-27632 D0-D5). However, those cells failed to express 
A

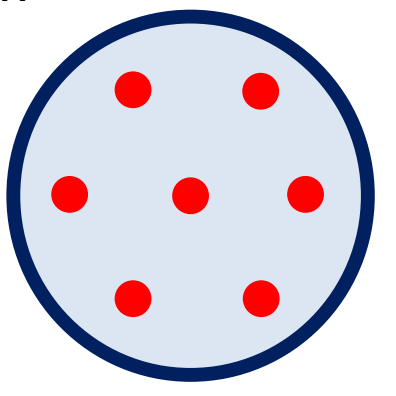

C

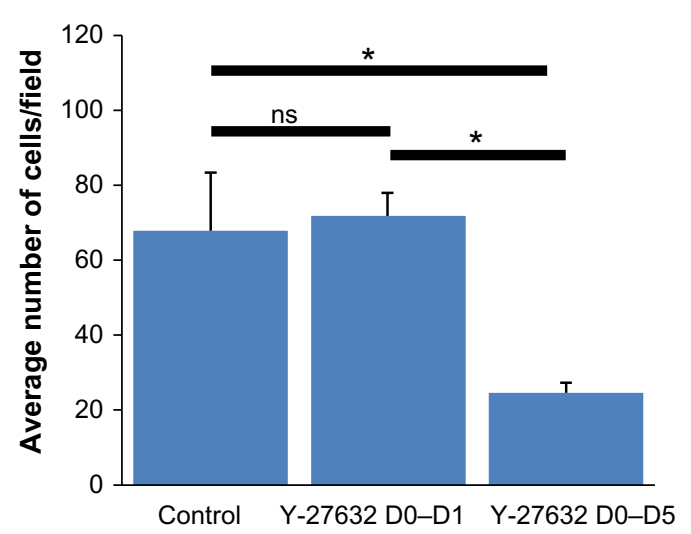

E

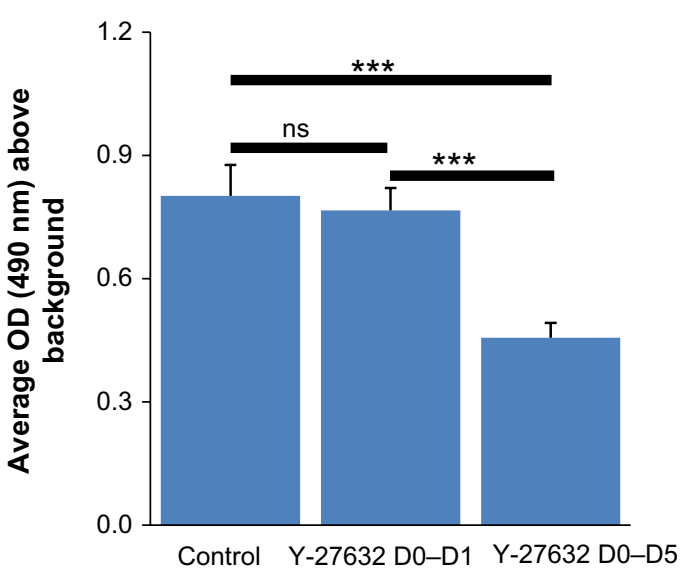

B

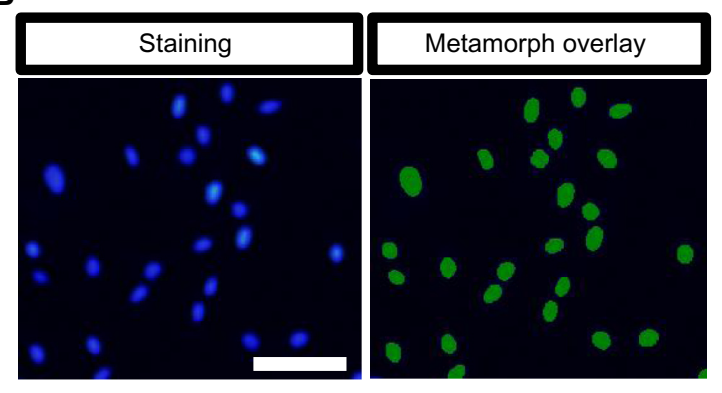

D

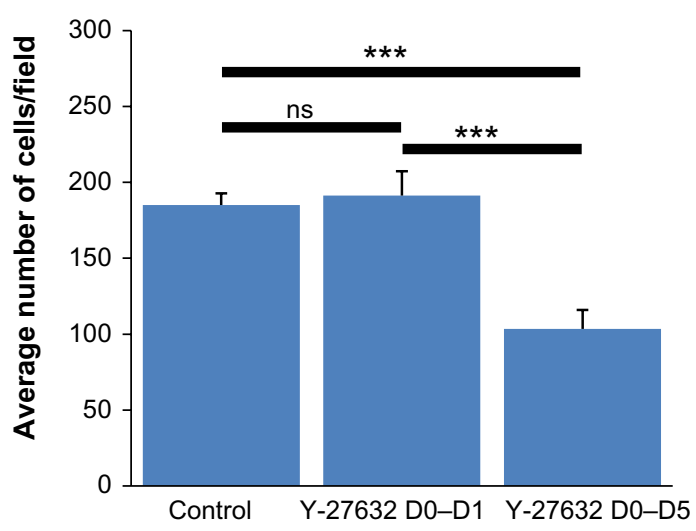

$\mathbf{F}$

MTS 5,000 cells $/ \mathrm{cm}^{2}$

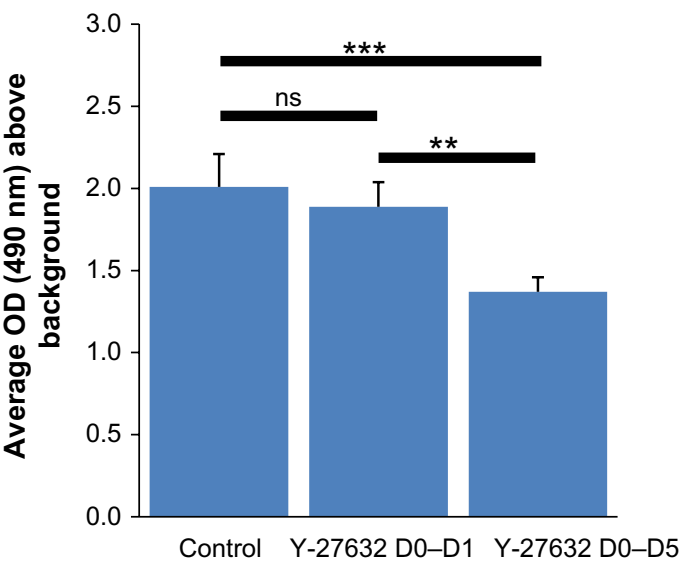

Figure 2 Cell number and cellular viability of hADSC cultures at day 5.

Notes: (A) Image acquisition for cell number quantification was performed in a blinded procedure through acquisition of images at I0X magnification in seven systematic regions (red dots) in each well studied. (B) Sample image of DAPI-stained hADSCs and corresponding overlay for automated quantitative determination of DAPI-positive nuclei using the Cell Scoring module of Metamorph NX software. Scale bar $=110 \mu \mathrm{m}$. (C) Continuous Y-27632 supplementation attenuated the expansion of hADSCs seeded initially at $I \times 10^{3}$ cells $/ \mathrm{cm}^{2}$ and (D) $5 \times 10^{3}$ cells $/ \mathrm{cm}^{2}$. Values shown as mean $\pm \mathrm{SEM}, \mathrm{n}=5$ ( $* * * P<0.00 \mathrm{I}, * P<0.05$, ns $=$ not significant). (E) The sustained treatment with $10 \mu M$ Y-27632 led to decreased cellular viability in hADSCs seeded initially at $I \times 10^{3}$ cells $/ \mathrm{cm}^{2}$, and (F) $5 \times 10^{3}$ cells/cm ${ }^{2}$ when compared to control conditions.Values shown as mean \pm $\mathrm{SEM}, \mathrm{n}=5$ (***P $<0.00 \mathrm{I}$, **P $<0.0 \mathrm{I}, \mathrm{ns}=$ not significant).

Abbreviations: DAPI, 4',6-diamidino-2-phenylindole; hADSCs, human adipose-derived stem cells; MTS, 3-(4,5-dimethylthiazol-2-yl)-5-(3-carboxymethoxyphenyl)-2-(4sulfonyl)- $2 \mathrm{H}$-tetrazolium; OD, optical density; SEM, standard error of the mean.

putative neuronal markers, namely the microtubule-associated protein 2 (MAP-2) as assessed by immunocytochemistry performed under optimized conditions in three independent experiments. Taken together, these results highlight that the continuous presence of Y-27632 in hADSC cultures could lead to decreased numbers of cells in culture when compared to control conditions, in contrast to what has been widely demonstrated in other stem cell types. 


\section{The effect of Y-27632 in hADSC cultures}

\section{is concentration-dependent}

To further confirm that the continuous presence of Y-27632 in culture does not enhance the expansion of hADSCs, we aimed to determine whether the effect of Y-27632 in hADSCs was concentration-dependent. Thus, we next performed studies involving serial concentrations of Y-27632 starting at $20 \mu \mathrm{M}$. For this set of experiments, hADSCs were seeded initially at 5,000 cells $/ \mathrm{cm}^{2}$ in 24 -well plates. Three replicate wells were used for each concentration tested. At the end of the optimized 5-day culture period, we observed a robust concentration-dependent decrease in hADSC numbers in cultures treated continuously with Y-27632 (Figure $3 \mathrm{~A})$. The calculated half maximal effective concentration $\left(\mathrm{EC}_{50}\right)$ for the continuous Y-27632 treatment was determined to be $\approx 7.97 \mu \mathrm{M}$. The decrease in cell numbers was statistically significant for the concentrations $10 \mu \mathrm{M}$ and $20 \mu \mathrm{M}$ (Figure 3A). These concentrations of Y-27632 were associated with significant increases in the average nuclear area of hADSCs (Figure 3B) and a significant decrease in the global cellular metabolic viability (Figure 3C). Together, our results not only demonstrate a robust Y-27632

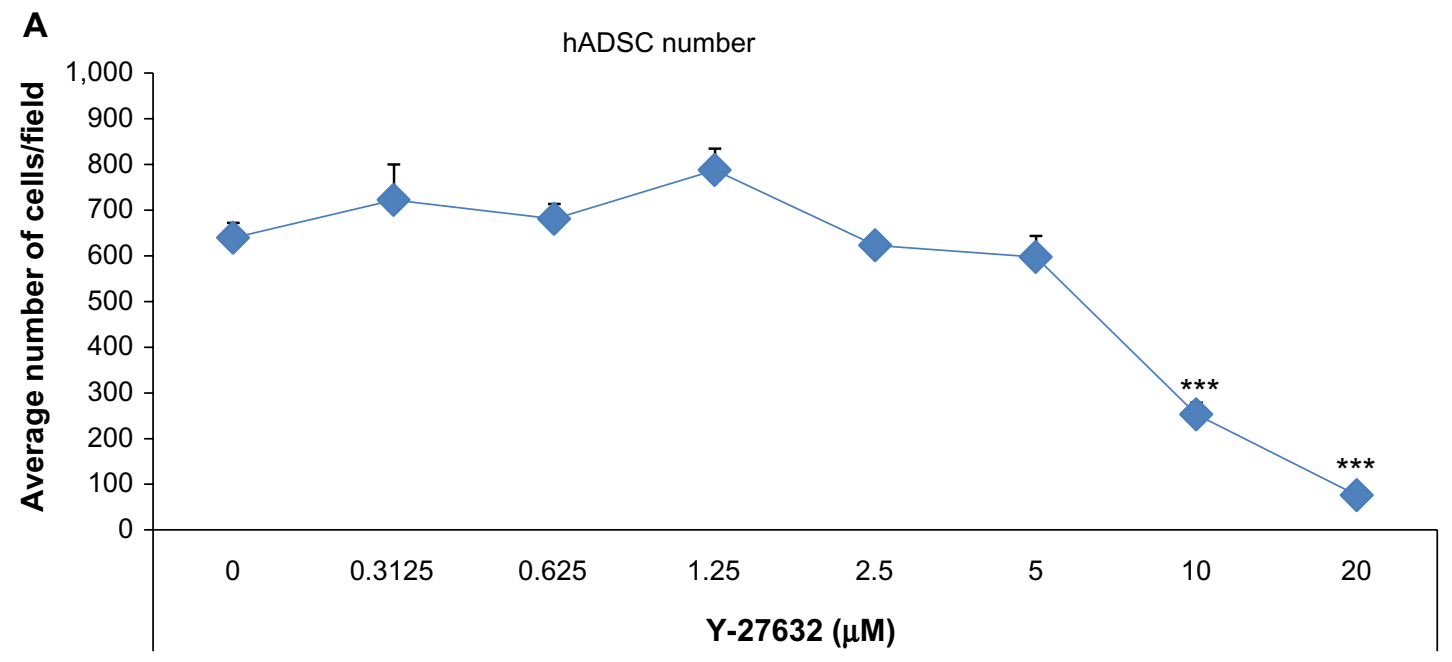

B

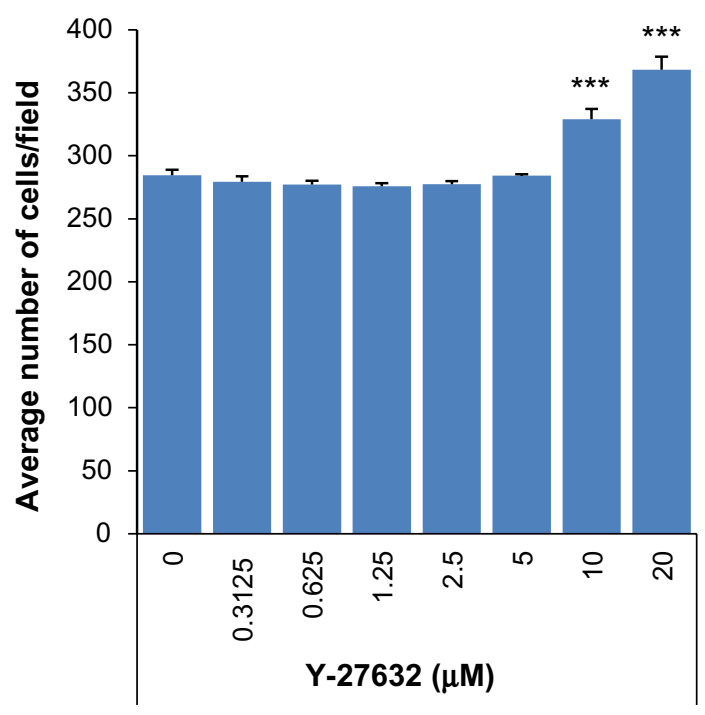

C

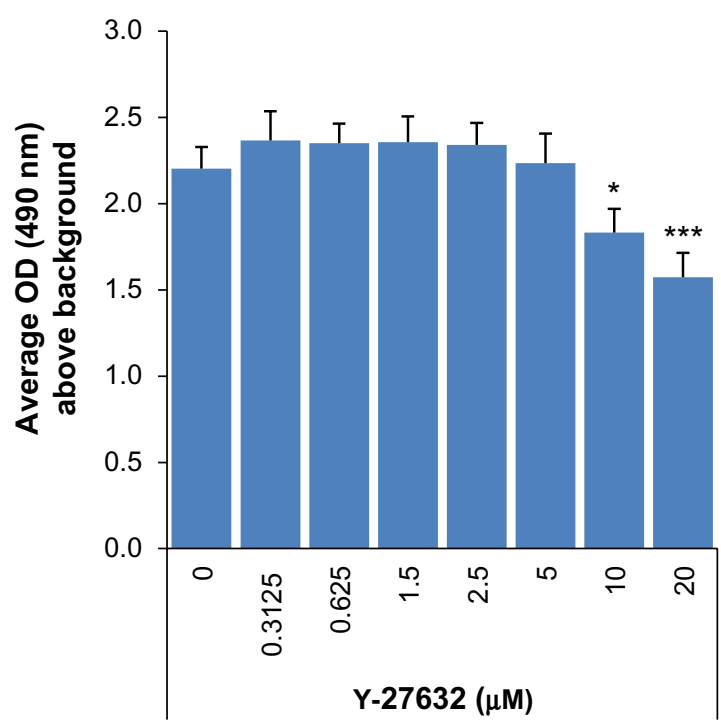

Figure 3 The effect of Y-27632 on the culture of hADSCs is concentration-dependent.

Notes: (A) Over 5 days, hADSCs were cultured under optimized conditions (without Y-27632) and in the presence of increasing concentrations of $Y$-27632. Cell numbers were significantly decreased when hADSCs were cultured with continuous $10 \mu \mathrm{M}$ or $20 \mu \mathrm{M} Y-27632$ supplementation. Values shown as mean $\pm \mathrm{SEM}$, $\mathrm{n}=5$ ( $* * * P<0.00 \mathrm{I})$. (B) hADSCs had significantly increased average nuclear area and (C) decreased global cell metabolic viability when cultured in the presence of $10 \mu M$ or $20 \mu M$ Y-27632. Values shown as mean $\pm \mathrm{SEM}, \mathrm{n}=5(* * * \mathrm{P}<0.001, * \mathrm{P}<0.05)$.

Abbreviations: hADSCs, human adipose-derived stem cells; MTS, 3-(4,5-dimethylthiazol-2-yl)-5-(3-carboxymethoxyphenyl)-2-(4-sulfonyl)-2H-tetrazolium; OD, optical density; SEM, standard error of the mean. 
concentration-dependent decrease in hADSC numbers, but also show for the first time that the standard $10 \mu \mathrm{M}$ concentration of Y-27632, commonly associated with pro-expansion effects in most stem cell types, does not enhance the culture of hADSCs.

\section{Effect of Y-27632 in the viability and continued culture of hADSCs}

The initial experiments which demonstrated that continuous Y-27632 supplementation is not beneficial for hADSC culture were performed using cells in passage $>6$. It is possible that at this later passage, cells have lost their multipotency and have become nonresponsive to the standard $10 \mu \mathrm{M}$ concentration of Y-27632. To rule out this possibility and to elucidate further the effect of Y-27632 in the hADSC cultures, we conducted a new set of experiments using hADSCs in earlier passages $(<\mathrm{p} 4)$. hADSCs were again grown in T75 flasks until confluency (Figure 4A). Afterwards, cells were trypsinized and the resultant cell suspension recovered to be replated at 5,000 cells $/ \mathrm{cm}^{2}$ in 6 -well plates. Cells were then allowed to expand over 5 days in the continuous presence or absence of the standard $10 \mu \mathrm{M}$ Y-27632. After 5 days, cells were trypsinized and counted with the help of a hematocytometer. Our data showed decreased numbers of cells in the wells treated with Y-27632 for 5 days (Figure 4B), which shows that the decrease in hADSC number upon Y-27632 treatment is also observed in earlier passages of hADSCs.

Numerous reports demonstrate that Y-27632 treatment is associated with enhanced survival of recently passaged cells. $^{7,8,24,25}$ To determine if Y-27632 is also able to enhance the survival of hADSCs after cell passaging, cells grown for 5 days in the presence or absence of Y-27632 were replated and cultured for the next 24 hours with or without Y-27632 (Figure 4A and C). After 24 hours, we registered similar numbers of hADSCs in the untreated and Y-27632-treated wells (Figure 4C; ns = not significant). This observation was present in cells either grown with or without Y-27632 in the previous 5 days. Together, these results show that Y-27632 is not able to significantly increase the survival of recently passaged hADSCs.

We next aimed to assess the effect of prolonged Y-27632 supplementation on the continuous proliferation and viability of hADSCs. Those hADSCs grown initially over 5 days in the presence or absence of Y-27632 were passaged and allowed to re-expand for an additional 5 days (Figure 4A and D). After passaging, each initial group of cells was subsequently submitted to treatment with or without Y-27632 (Figure 4A and D). Therefore, hADSCs were grown under four different medium conditions (Figure 4A): 10 days of growth in control conditions $(\mathrm{C} / \mathrm{C}) ; 5$ days of growth in control conditions plus 5 days of continuous Y-27632 supplementation (C/Y); 5 days of growth with Y-27632 followed by growth in control conditions (Y/C); and 10 days of growth with Y-27632 treatment (Y/Y). We observed a continuous increase in the number of hADSCs in culture in both the first and the second period of 5 days of cell culture across the different growth conditions, which shows that Y-27632 treatment does not block the proliferation of hADSCs (Figure 4C vs 4D). After 10 days of culture, the wells submitted to a continuous treatment with Y-27632 during the second growth period had the lowest number of hADSCs (C/Y and $\mathrm{Y} / \mathrm{Y}$; Figure 4D). Contrarily, cells grown under control conditions in both periods had the greatest number of hADSCs in culture (C/C; Figure 4D). Interestingly, in the wells submitted to an initial 5-day treatment with Y-27632 and then a second period of growth in control conditions (Y/C), the number of hADSCs present in culture was similar to growth in control conditions for the 10 days (C/C; Figure 4D). In line with this, the global metabolic viability of hADSCs was decreased in the wells treated with Y-27632 for the last 5 days of cell growth (C/Y and Y/Y; Figure 4E). Yet, we did not register significant differences in the global levels of cell proliferation among the four growth conditions studied (Figure 4F). Together, this data reveals a long-term decrease in the number of hADSCs in the presence of Y-27632 when compared to control conditions. Our results also suggest that the removal of the drug from the growth medium allows cultures to re-expand up to the levels similar to that of the control growth condition, implying that the effects of Y-27632 on hADSC cultures are not irreversible, but seem to depend on the continuous presence of the drug.

The previous experiments were performed under a paradigm of continuous Y-27632 supplementation. To further confirm that the decrease in the number of hADSCs pertained by Y-27632 depends on continuous drug supplementation, we performed the same experiments under a paradigm of transient Y-27632 treatment during the 24 hours prior to cell passaging (Figure 5A). We observed that the 24 hours of treatment did not have a significant impact on the total number of cells retrieved from the wells after 5 days of hADSC expansion, resulting in similar total cell yields when compared to the control conditions (Figure 5A and 5B). In line with previous data, 24 hours after hADSC passaging, we registered similar numbers of hADSCs in the untreated and Y-27632-treated wells (Figure 5C), further confirming that Y-27632 does not seem to significantly enhance the survival of hADSCs. The hADSCs 
A

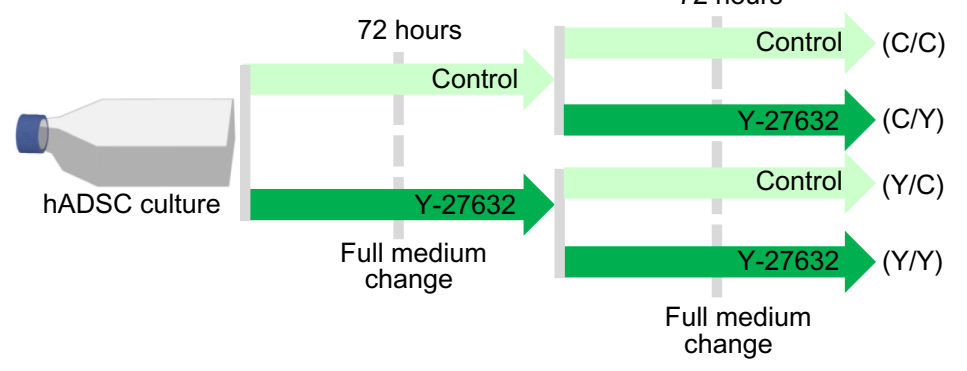

B

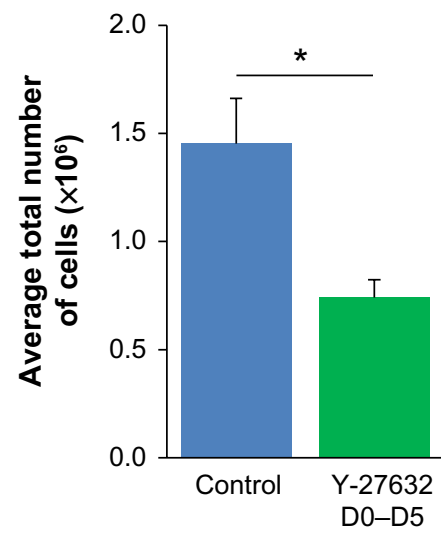

C hADSC cell number 24 hours post-passaging

D Total hADSC cell number at day $5+5$
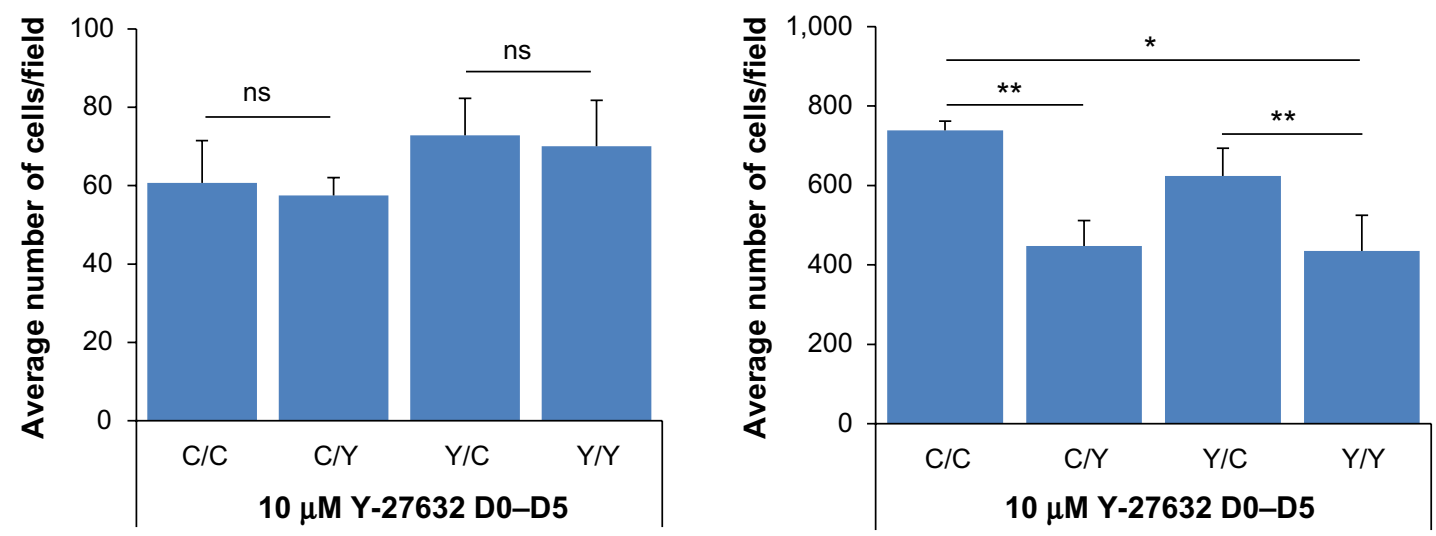

E

MTS

$\mathbf{F}$

BrdU
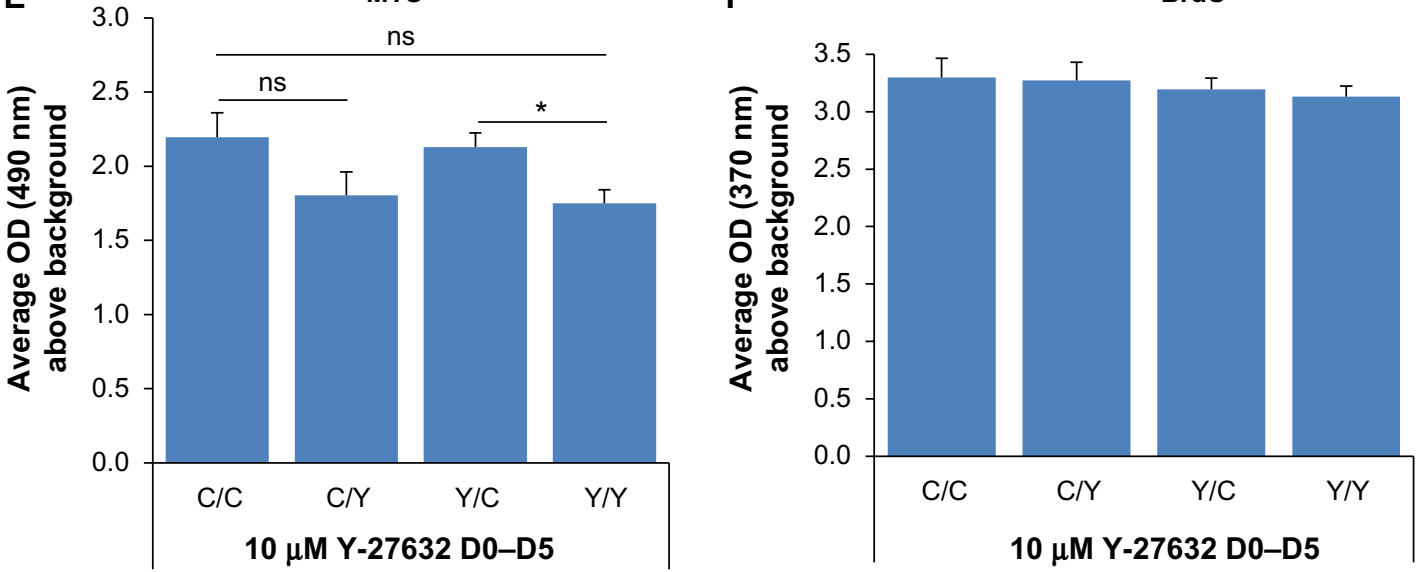

Figure 4 Prolonged Y-27632 supplementation is not associated with improvements in the expansion and survival of hADSCs.

Notes: (A) Diagram of the protocol used to study the effect of prolonged Y-27632 supplementation in early passage hADSC cultures. hADSCs were grown initially over 5 days in the presence or absence of Y-27632. Afterwards, cells were passaged and allowed to grow for an additional 5 days. The hADSCs from the two initial groups were each grown subsequently with or without Y-27632. (B) At the end of the initial 5 days of culture, continuous Y-27632 treatment led to systematically decreased numbers of hADSCs, even at early passages of hADSCs. Values shown as mean \pm SEM, $n=5(* P<0.05)$. (C) Twenty-four hours after passaging, $Y$-27632 supplementation did not promote enhanced survival of hADSCs. This result was observed whether previous 5-day treatment with $\mathrm{Y}-27632$ was present or absent. Values shown as mean $\pm \mathrm{SEM}$, $\mathrm{n}=4$ ( $\mathrm{ns}=$ not significant). (D) After 10 days of cell culture, hADSC numbers were significantly decreased in wells grown continuously with Y-27632 for the previous 5 days. An initial exposure to Y-27632 for 5 days followed by growth in control conditions did not preclude hADSCs to re-expand up to levels similar to the control conditions. Values shown as mean \pm SEM, $n=5(* * P<0.0$ I, $* P<0.05)$. (E) The continuous presence of $Y-27632$ was associated with decreased global cellular metabolic viability of hADSCs after I0 days of culture. Values shown as mean \pm SEM, $n=5(* P<0.05$, ns $=$ not significant). (F) Global hADSC proliferation remained apparently unaffected by the presence of $Y-27632$, which is in line with the observed increase in cell numbers in both 5-day periods of cell culture studied. Values shown as mean \pm SEM, $n=3$.

Abbreviations: BrdU, 5-bromo-2'-deoxyuridine; hADSCs, human adipose-derived stem cells; MTS, 3-(4,5-dimethylthiazol-2-yl)-5-(3-carboxymethoxyphenyl)-2-(4-sulfonyl)$2 \mathrm{H}$-tetrazolium; OD, optical density; SEM, standard error of the mean; C, control; Y, Y-27632. 
A

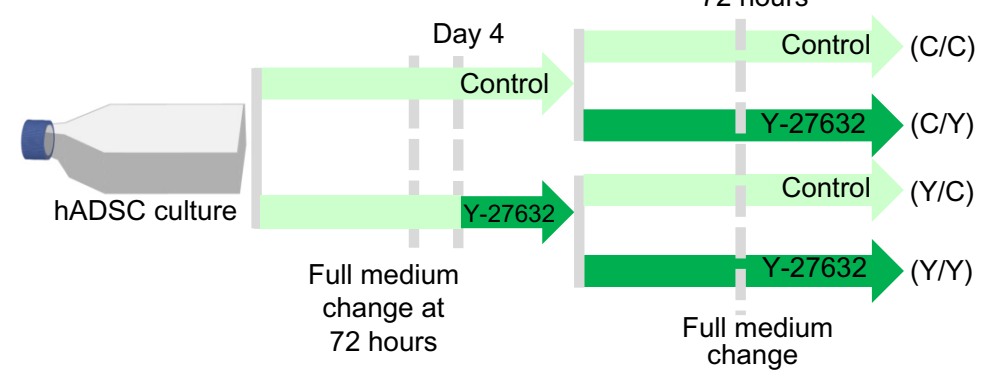

\section{5 days}

Control growth medium

Y-27632-supplemented growth medium

C

hADSC cell number 24 hours post-passaging

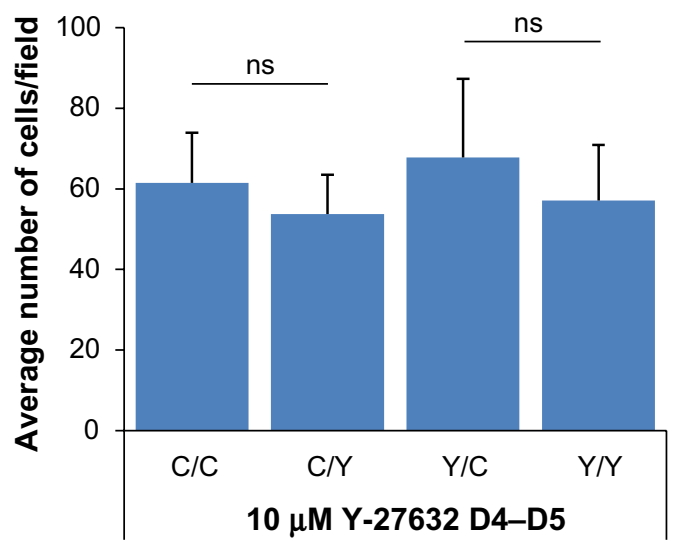

E

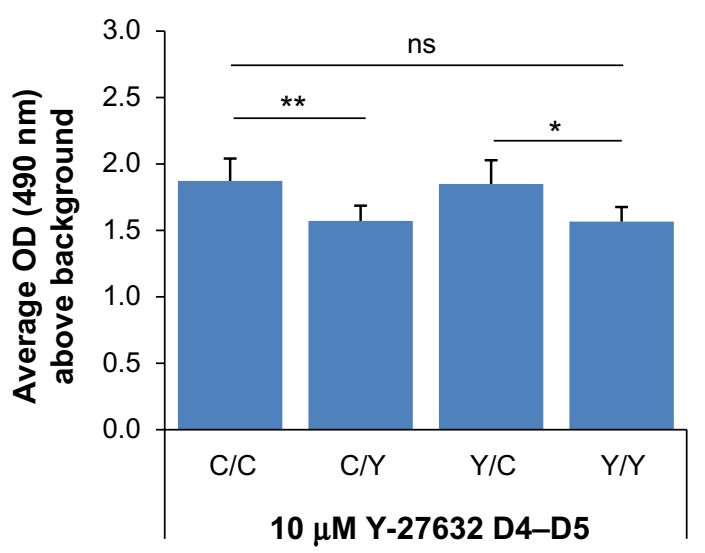

$\mathbf{F}$
B Total hADSC number day 5

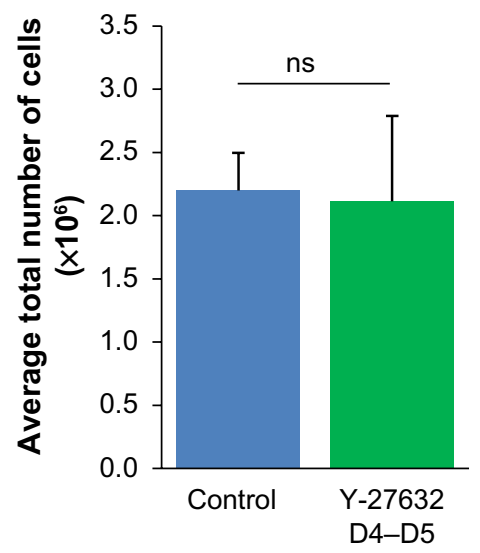

Total hADSC cell number at day $5+5$

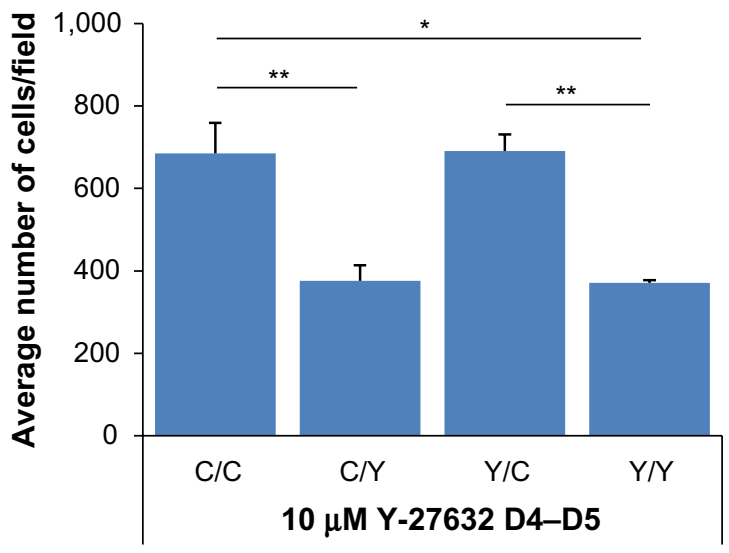

BrdU

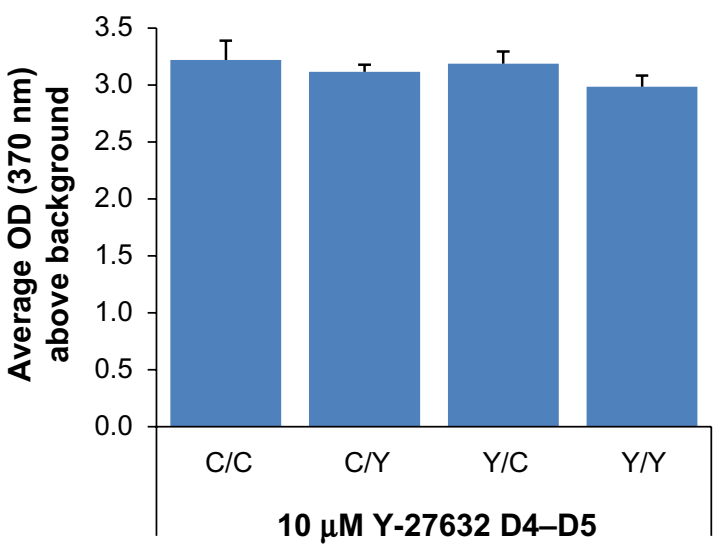

Figure 5 Y-27632 exposure over 24 hours prior to cell passaging was not associated with improvements in the expansion and survival of hADSCs.

Notes: (A) Diagram of the protocol used to study the effect of Y-27632 supplementation 24 hours before passaging in hADSC cultures. (B) At the end of the initial 5 days of culture, transient 24 -hour exposure of hADSCs to Y-27632 treatment did not alter the number of cells retrieved upon passaging at day 5 . Values shown as mean \pm SEM, $n=5$ (ns = not significant). (C) Twenty-four hours after passaging, Y-27632 supplementation was not linked to enhancements in the survival of hADSCs. This result was observed whether treatment with Y-27632 in the preceding 24 hours was present or absent. Values shown as mean \pm SEM, $n=4$ (ns = not significant). (D) After I0 days of cell culture, hADSC numbers were significantly decreased in wells grown continuously with Y-27632 for the previous 5 days. An initial exposure to Y-27632 for 24 hours followed by growth in control conditions did not preclude hADSCs to expand up to levels similar to the control conditions. Values shown as mean $\pm \mathrm{SEM}$, $\mathrm{n}=5$ ( $* * P<0.0 \mathrm{I}, * \mathrm{P}<0.05$ ). (E) Continuous Y-27632 supplementation was associated with decreased global cellular metabolic viability of hADSCs after I0 days of culture. Values shown as mean \pm SEM, $\mathrm{n}=5$ ( $* * \mathrm{P}<0.01, * \mathrm{P}<0.05$, ns = not significant). (F) Global hADSC proliferation levels in the presence of $Y-27632$ were similar to control conditions, which is in line with the observed increase in cell numbers in both 5-day periods of cell cultures studied for control and $\mathrm{Y}$-27632-treated wells. Values shown as mean $\pm \mathrm{SEM}$, $\mathrm{n}=3$.

Abbreviations: BrdU, 5-bromo-2'-deoxyuridine; hADSCs, human adipose-derived stem cells; MTS, 3-(4,5-dimethylthiazol-2-yl)-5-(3-carboxymethoxyphenyl)-2-(4-sulfonyl)$2 \mathrm{H}$-tetrazolium; OD, optical density; SEM, standard error of the mean; C, control; Y, Y-27632. 
exposed to Y-27632 over the 24 hours before passaging were also replated and allowed to cultivate for an additional 5 days with and without Y-27632 (Figure 5A and 5D). As a result, the cells were again studied in four different medium conditions (Figure 5A): 10 days of culture in control conditions (C/C); 5 days of growth in control conditions plus 5 days of continuous Y-27632 supplementation (C/Y); 4 days of growth in control conditions followed by a 24-hour exposure to Y-27632; and then either growth in control conditions (Y/C) or 5-day growth submitted to Y-27632 (Y/Y). Here, we also observed a continuous increase in the number of hADSCs in culture in both the first and the second period of 5 days of cell culture across the different growth conditions (Figure 5C vs 5D). After 10 days of culture, the wells submitted to Y-27632 treatment during the second growth period had the lowest number of hADSCs (Figure 5D) and cells grown under control conditions for the entire 10-day period had the greatest number of hADSCs in culture (Figure 5D). Interestingly, in the wells where hADSCs were submitted to an initial 24 hours of Y-27632 treatment and then a second period of growth in control conditions (Y/C), the number of hADSCs present in culture was similar to growth in control conditions for the 10 days $(\mathrm{C} / \mathrm{C}$; Figure $5 \mathrm{D})$. The global metabolic viability of hADSCs was decreased in the wells continuously treated with Y-27632 for the last 5 days of cell growth (Figure 5E). Interestingly, we did not register significant differences in the levels of cell proliferation among the four medium combinations tested (Figure 5F). This data further confirms that the continued decrease in the number of hADSCs in the presence of Y-27632 depends on the constant presence of the drug.

Taken together, the data reported in the present study demonstrate for the first time that continuous supplementation of hADSC cultures with Y-27632, even at the standard $10 \mu \mathrm{M}$ concentration, does not constitute a beneficial strategy to improve the culture of hADSCs.

\section{Discussion}

The continuous addition of Y-27632 to stem cell cultures has been linked to considerable improvements in the expansion of some stem cell populations, specifically hPSCs and hNPCs. ${ }^{6,23,26-28}$ We showed here that growing hADSC cultures in the presence of Y-27632 did not add benefits in terms of cell numbers and cell viability; moreover, the continuous presence of Y-27632 was shown to be detrimental for the expansion of this population of stem cells given that cell numbers and cell metabolic viability were significantly decreased in the different paradigms studied when compared to control conditions. However, the decrease in the number of hADSCs upon continuous treatment with Y-27632 was not linked to a block in cell proliferation, as cell numbers increased during 5 days and global BrdU levels were similar in wells treated with or without Y-27632. In addition, the removal of the Y-27632 compound from the medium allowed cells previously exposed to Y-27632 treatment (either 24 hours or 5 days continuously) to expand up to the levels of the control condition, which implies that the effects of Y-27632 on hADSC cultures reported here are not irreversible, but seem to depend on the continuous presence of the drug. ${ }^{29}$ Therefore, it is plausible that Y-27632 is lengthening the progression of the dividing hADSCs through the cell cycle, which could explain overall lower hADSC numbers in comparison to control conditions, despite similar global levels of BrdU in the 48-hour pulse performed. Our results are in line with previous studies showing that the expansion of $\mathrm{CD} 34^{+}$hematopoietic progenitor cells is impaired in the presence of Y-27632. ${ }^{30,31}$ Both hADSCs and $\mathrm{CD} 4^{+}$hematopoietic progenitor cells are adult stem cells of mesodermal origin and Y-27632 could be decreasing their expansion rates by using a common mechanism. The present study also showed that the application of Y-27632 to recently passaged hADSC cultures did not significantly increase the number of hADSCs 24 hours after passaging when compared to control conditions. This result is in contrast with studies involving other stem cells types, namely hPSCs, in which this compound is known to significantly increase cell survival of those stem cells upon dissociation due to its well-known antiapoptotic effects. ${ }^{27,28}$ It is possible that the proapoptotic mechanisms activated in hPSCs upon dissociation differ from the possible proapoptotic mechanisms present in hADSCs in the first 24 hours after cell passaging. On the other hand, the molecular inhibition pertained by this compound in hADSCs (mesenchymal lineage) might lead to a diverse cellular phenotype from what has been widely described for hPSCs. Future studies are needed to thoroughly address the cellular mechanisms responsible for the diverse responses of different stem cell types to Y-27632.

The great majority of studies in the literature that demonstrate the beneficial effects of Y-27632 for cell proliferation and survival have been obtained using a standard $10 \mu \mathrm{M}$ concentration of Y-27632. Here, we studied different concentrations of Y-27632 and showed that in wells grown continuously under $10 \mu \mathrm{M}$ or $20 \mu \mathrm{M}$ of Y-27632, there are significantly decreased numbers of hADSCs. Previous in vitro studies involving Y-27632 have established that this compound at $10 \mu \mathrm{M}$ concentration, besides ROCK, can in addition strongly inhibit other kinases, namely PRK2, mitogen- and stress-activated protein kinase-1 (MSK1), leucine-rich repeat 
protein kinase-2 (LRRK2), and MNK1. ${ }^{32,33}$ This might explain the observed decrease in the number of viable cells at day 5 upon continuous Y-27632 supplementation, since the sustained inhibition of several protein kinases might be detrimental to cellular homeostasis in the long run.

A recent report in the literature has proposed that hADSCs treated with Y-27632 develop morphological changes resembling neuron-like cells. ${ }^{34}$ Here, the continuous presence of Y-27632 led to the appearance of hADSCs with similar neuron-like shapes. We could demonstrate that these cells fail to express putative neuronal markers, namely MAP-2. Yet, it remains to be confirmed that Y-27632 alters the lineage commitment potential of hADSCs. Eventually, these morphological changes add another layer of evidence to highlight the negative value of adding Y-27632 to hADSC cultures, if the purpose is to culture this type of adult stem cells more efficiently.

\section{Conclusion}

The present work shows that Y-27632 supplementation not only does not increase the plating efficiency of hADSCs, but also fails to enhance the expansion of this type of human adult stem cells. In contrast to other stem cell types, Y-27632 supplementation does not seem to constitute a successful strategy to improve hADSC cultures.

\section{Acknowledgments}

We thank Jeffrey M Gimble (Center for Stem Cell Research and Regenerative Medicine, Tulane University and LaCell LLC, New Orleans, LA, USA) for kindly isolating, characterizing, and sharing the cellular lines of hADSCs used in the present study and for critical input on the manuscript. We also would very much like to thank Laurent Roybon (Stem Cell Laboratory for CNS Disease Modeling, Department of Experimental Medical Science, Lund University, Lund, Sweden) for the utilization of Metamorph NX software for automated cell quantification. We are grateful to Miguel Carvalho, Ana Pires, Eduardo Gomes, Fábio Teixeira and Nuno Silva for technical assistance. This work was supported by the Portuguese Foundation for Science and Technology (predoctoral fellowship to NJL [SFRH/BD/33421/2008]; FCT Investigator Program to AJS) and the Luso-American Development Foundation.

\section{Author contributions}

NJL: collection of data, conception and design, data analysis and interpretation, manuscript writing; SCS: collection of data, data analysis and interpretation, manuscript writing; AJS: conception and design, data analysis and interpretation, manuscript writing; NS: conception and design, data analysis and interpretation, manuscript writing. All authors read and approved the final manuscript.

\section{Disclosure}

The authors report no conflicts of interest in this work.

\section{References}

1. Evans M. Discovering pluripotency: 30 years of mouse embryonic stem cells. Nature reviews. Nat Rev Mol Cell Biol. 2011;12(10):680-686.

2. Gokhale PJ, Andrews PW. Human embryonic stem cells: 10 years on. Lab Invest. 2009;89(3):259-262.

3. Kørbling M, Estrov Z. Adult stem cells for tissue repair - a new therapeutic concept? N Engl J Med. 2003;349(6):570-582.

4. Valamehr B, Tsutsui H, Ho CM, Wu H. Developing defined culture systems for human pluripotent stem cells. Regen Med. 2011;6(5):623-634.

5. Villa-Diaz LG, Ross AM, Lahann J, Krebsbach PH. Concise review: the evolution of human pluripotent stem cell culture: from feeder cells to synthetic coatings. Stem Cells. 2013;31(1):1-7.

6. Kurosawa H. Application of Rho-associated protein kinase (ROCK) inhibitor to human pluripotent stem cells. J Biosci Bioeng. 2012;114(6):577-581.

7. Watanabe K, Ueno M, Kamiya D, et al. A ROCK inhibitor permits survival of dissociated human embryonic stem cells. Nat Biotechnol. 2007;25(6):681-686.

8. Pakzad M, Totonchi M, Taei A, Seifinejad A, Hassani SN, Baharvand $\mathrm{H}$. Presence of a ROCK inhibitor in extracellular matrix supports more undifferentiated growth of feeder-free human embryonic and induced pluripotent stem cells upon passaging. Stem Cell Rev. 2010;6(1):96-107.

9. Gauthaman K, Fong CY, Bongso A. Effect of ROCK inhibitor Y-27632 on normal and variant human embryonic stem cells (hESCs) in vitro: its benefits in hESC expansion. Stem Cell Rev. 2010;6(1):86-95.

10. Li X, Meng G, Krawetz R, Liu S, Rancourt DE. The ROCK inhibitor Y-27632 enhances the survival rate of human embryonic stem cells following cryopreservation. Stem Cells Dev. 2008; 17(6):1079-1085.

11. Gimble J, Guilak F. Adipose-derived adult stem cells: isolation, characterization, and differentiation potential. Cytotherapy. 2003;5(5):362-369.

12. Gimble JM, Katz AJ, Bunnell BA. Adipose-derived stem cells for regenerative medicine. Circ Res. 2007;100(9):1249-1260.

13. Bunnell BA, Estes BT, Guilak F, Gimble JM. Differentiation of adipose stem cells. Methods Mol Biol. 2008;456:155-171.

14. Lindroos B, Suuronen R, Miettinen S. The potential of adipose stem cells in regenerative medicine. Stem Cell Rev. 2011;7(2):269-291.

15. Aguena M, Fanganiello RD, Tissiani LA, et al. Optimization of parameters for a more efficient use of adipose-derived stem cells in regenerative medicine therapies. Stem Cells Int. 2012;2012:303610.

16. Mizuno H, Tobita M, Uysal AC. Concise review: adipose-derived stem cells as a novel tool for future regenerative medicine. Stem Cells. 2012;30(5):804-810.

17. Salgado AJ, Reis RL, Sousa NJ, Gimble JM. Adipose tissue derived stem cells secretome: soluble factors and their roles in regenerative medicine. Curr Stem Cell Res Ther. 2010;5(2):103-110.

18. Dubois SG, Floyd EZ, Zvonic S, et al. Isolation of human adiposederived stem cells from biopsies and liposuction specimens. Methods Mol Biol. 2008;449:69-79.

19. Nakamura K, Yoshimura A, Kaneko T, Sato K, Hara Y. ROCK inhibitor Y-27632 maintains the proliferation of confluent human mesenchymal stem cells. J Periodontal Res. 2014;49(3):363-370.

20. Claassen DA, Desler MM, Rizzino A. ROCK inhibition enhances the recovery and growth of cryopreserved human embryonic stem cells and human induced pluripotent stem cells. Mol Reprod Dev. 2009;76(8):722-732. 
21. Heng BC. Effect of Rho-associated kinase (ROCK) inhibitor Y-27632 on the post-thaw viability of cryopreserved human bone marrow-derived mesenchymal stem cells. Tissue Cell. 2009;41(5):376-380.

22. Li X, Krawetz R, Liu S, Meng G, Rancourt DE. ROCK inhibitor improves survival of cryopreserved serum/feeder-free single human embryonic stem cells. Hum Reprod. 2009;24(3):580-589.

23. Rungsiwiwut R, Manolertthewan C, Numchaisrika P, et al. The ROCK inhibitor Y-26732 enhances the survival and proliferation of human embryonic stem cell-derived neural progenitor cells upon dissociation. Cells Tissues Organs. 2013;198(2):127-138.

24. Zhang L, Valdez JM, Zhang B, Wei L, Chang J, Xin L. ROCK inhibitor Y-27632 suppresses dissociation-induced apoptosis of murine prostate stem/progenitor cells and increases their cloning efficiency. PLoS One. 2011;6(3):e18271.

25. Ramasamy TS, Yu JS, Selden C, Hodgson H, Cui W. Application of three-dimensional culture conditions to human embryonic stem cellderived definitive endoderm cells enhances hepatocyte differentiation and functionality. Tissue Eng Part A. 2013;19(3-4):360-367.

26. Ohgushi M, Sasai Y. Lonely death dance of human pluripotent stem cells: ROCKing between metastable cell states. Trends Cell Biol. 2011;21(5):274-282.

27. Ohgushi M, Matsumura M, Eiraku M, et al. Molecular pathway and cell state responsible for dissociation-induced apoptosis in human pluripotent stem cells. Cell Stem Cell. 2010;7(2):225-239.

28. Chen G, Hou Z, Gulbranson DR, Thomson JA. Actin-myosin contractility is responsible for the reduced viability of dissociated human embryonic stem cells. Cell Stem Cell. 2010;7(2):240-248.
29. Chapman S, McDermott DH, Shen K, Jang MK, McBride AA. The effect of Rho kinase inhibition on long-term keratinocyte proliferation is rapid and conditional. Stem Cell Res Ther. 2014;5(2):60.

30. Bueno C, Montes R, Menendez P. The ROCK inhibitor Y-27632 negatively affects the expansion/survival of both fresh and cryopreserved cord blood-derived CD34+ hematopoietic progenitor cells: Y-27632 negatively affects the expansion/survival of CD34+HSPCs. Stem Cell Rev. 2010;6(2):215-223.

31. Burthem J, Rees-Unwin K, Mottram R, et al. The rho-kinase inhibitors Y-27632 and fasudil act synergistically with imatinib to inhibit the expansion of ex vivo CD34(+) CML progenitor cells. Leukemia. 2007;21(8):1708-1714.

32. Davies SP, Reddy H, Caivano M, Cohen P. Specificity and mechanism of action of some commonly used protein kinase inhibitors. Biochem J. 2000;351(Pt 1):95-105.

33. Nichols RJ, Dzamko N, Hutti JE, et al. Substrate specificity and inhibitors of LRRK2, a protein kinase mutated in Parkinson's disease. Biochem J. 2009;424(1):47-60.

34. Xue ZW, Shang XM, Xu H, et al. Rho-associated coiled kinase inhibitor Y-27632 promotes neuronal-like differentiation of adult human adipose tissue-derived stem cells. Chin Med J (Engl). 2012;125(18):3332-3335.
Stem Cells and Cloning: Advances and Applications

\section{Publish your work in this journal}

Stem Cells and Cloning: Advances and Applications is an international, peer-reviewed, open access journal. Areas of interest in stem cell research include: Embryonic stem cells; Adult stem cells; Blastocysts; Cordblood stem cells; Stem cell transformation and culture; Therapeutic cloning; Umbilical cord blood and bone marrow cells; Laboratory,

\section{Dovepress}

animal and human therapeutic studies; Philosophical and ethical issues related to stem cell research. This journal is indexed on CAS. The manuscript management system is completely online and includes a quick and fair peer-review system. Visit http://www.dovepress.com/ testimonials.php to read real quotes from published authors. 